\title{
Distributed Control of Multi-zone HVAC Systems Considering Indoor Air Quality
}

\author{
Yu Yang, Student Member, IEEE, Seshadhri Srinivasan, Senior Member, IEEE, \\ Guoqiang Hu, Senior Member, IEEE, and Costas J. Spanos, Fellow, IEEE
}

\begin{abstract}
This paper studies a scalable control method for multi-zone heating, ventilation and air-conditioning (HVAC) systems to optimize the energy cost for maintaining thermal comfort and indoor air quality (IAQ) (represented by $\mathrm{CO}_{2}$ ) simultaneously. This problem is computationally challenging due to the complex system dynamics, various spatial and temporal couplings as well as multiple control variables to be coordinated. To address the challenges, we propose a two-level distributed method (TLDM) with a upper level and lower level control integrated. The upper level computes zone mass flow rates for maintaining zone thermal comfort with minimal energy cost, and then the lower level strategically regulates zone mass flow rates and the ventilation rate to achieve IAQ while preserving the near energy saving performance of upper level. As both the upper and lower level computation are deployed in a distributed manner, the proposed method is scalable and computationally efficient. The near-optimal performance of the method in energy cost saving is demonstrated through comparison with the centralized method. In addition, the comparisons with the existing distributed method show that our method can provide IAQ with only little increase of energy cost while the latter fails. Moreover, we demonstrate our method outperforms the demand controlled ventilation strategies (DCVs) for IAQ management with about $8-10 \%$ energy cost reduction.
\end{abstract}

Note to Practitioners-The high portion of building energy consumption has motivated the energy saving for HVAC systems. Concurrently, the living standards for indoor environment are rising among the occupants. Nevertheless, the status quo on improving building energy efficiency has mostly focused on maintaining thermal comfort (such as temperature), and the indoor air quality (IAQ) (usually represented by $\mathrm{CO}_{2}$ level) has been seldom incorporated.

In our previous work with the similar setting, we observed that the $\mathrm{CO}_{2}$ levels will surge beyond tolerance during the high occupancy periods if only thermal comfort is considered for HVAC control. This deduces the IAQ and thermal comfort should be jointly considered while pursuing the energy cost saving target and thus studied in this paper. This task is computationally cumbersome due to the complex system dynamics (thermal and $\mathrm{CO}_{2}$ ) and tight correlations among the different control components (variable air volume and fresh air damper). To cope

This work was supported by the Republic of Singapore's National Research Foundation through a grant to the Berkeley Education Alliance for Research in Singapore (BEARS) for the Singapore-Berkeley Building Efficiency and Sustainability in the Tropics (SinBerBEST) Program. BEARS has been established by the University of California, Berkeley as a center for intellectual excellence in research and education in Singapore.

Yu Yang and Seshadhri Srinivasan are with SinBerBEST, Berkeley Education Alliance for Research in Singapore, Singapore, 138602. E-mail: ( \{yu.yang, seshadhri.srinivasan\}@bears-berkeley.sg).

Guoqiang $\mathrm{Hu}$ is with the School of Electrical and Electronic Engineering, Nanyang Technological University, Singapore, 639798. E-mail: (gqhu@ntu.edu.sg).

Costas J. Spanos is with the Department of Electrical Engineering and Computer Sciences, University of California, Berkeley, CA, 94720 USA. Email: (spanos@berkeley.edu) with these challenges, this work develops a two-level distributed computation paradigm for HVAC systems based on problem structures. Specifically, the upper level control first calculates zone mass flow rates for maintaining comfortable zone temperature with minimal energy cost and then the lower level strategically regulates the computed zone mass flow rates as well as ventilation rate to satisfy IAQ while preserving the near energy saving performance of the upper level control. As both the upper and lower level calculation can be implemented in a distributed manner, the proposed method is scalable to large multi-zone deployment. The method's performance both in maintaining comfort (i.e., thermal comfort and IAQ) and energy cost saving is demonstrated via simulations in comparisons with the centralized method, the distributed token-based scheduling strategy and the demand controlled ventilation strategies.

Index Terms-multi-zone HVAC system, two-level, distributed approach, IAQ, $\mathrm{CO}_{2}$.

\section{INTRODUCTION}

The heating, ventilation and air-conditioning (HVAC) systems account for $40 \%-50 \%$ of building energy consumption for maintaining comfortable indoor environment [1]. Aware of the high portion of energy consumption, the proposition to improve energy efficiency for HVAC systems has stimulated widespread attention [2, 3]. A plenty of works have demonstrated substantial energy can be saved by deploying advanced HVAC control strategies (see [4, 5], for examples). However, regarding the human sensation, the main focus has been placed on thermal comfort which is usually indicated by temperature and humidity $[6,7]$, the indoor air quality (IAQ), such as the carbon dioxide $\left(\mathrm{CO}_{2}\right)$ concentration has been seldom studied.

IAQ is closely related to the mechanical ventilation rate (i.e., fresh air infusion) of HVAC systems, especially nowadays where most buildings are constructed in closed envelope for energy saving concerns. IAQ has emerged as a critical issue along developing building automation. On one hand, the living standards and awareness of health are rising among the occupants; on the other hand, IAQ is closely related to occupant well-being and working productivity [8,9]. A study conducted by the National Institute of Environmental Health Science (NIEHS) in 2015 has demonstrated the impact of IAQ on cognitive abilities [10]. Significantly, the findings show that the strategic skills of occupants reduce to $20 \%$ with an indoor $\mathrm{CO}_{2}$ concentration $1,400 \mathrm{ppm}$ compared to the normal outdoor level of $400 \mathrm{ppm}$. Straightforwardly, for energy-efficient HVAC control, as the thermal comfort only relates to zone mas flow rates, the fresh air ventilation of HVAC systems is usually suppressed to the lower boundary 
to minimize the cooling load. However, for IAQ management, sufficient ventilation is required to dilute the indoor air. This implies considering thermal comfort alone is not enough for developing energy-efficient HVAC control strategies. As in such setting, the energy saving target may be achieved at the expense of awful IAQ. Specifically, we can imagine that the $\mathrm{CO}_{2}$ concentration will accumulate and surge beyond tolerance during the time periods with high occupancy though the temperature is maintained comfortable if sufficient fresh air ventilation is not provided by the HVAC systems.

Notably, increasing focus has been shifted towards IAQ management for HVAC systems both from industries and research communities in recent years. Some industries have informed that the thermostats will integrate $\mathrm{CO} 2$ sensors inside for monitoring IAQ in foreseeable future [11]. Within the research communities, some pioneering works have jointly considered IAQ and thermal comfort while studying energyefficient HVAC control (see [12-14], for examples). Nevertheless, this problem hasn't been well addressed yet as i) the related works are still fairly limited (see the references therein); and ii) most of them are for single-zone cases $[12,13]$ or not scalable to multi-zone commercial buildings due to the centralized computation framework [14]. The computational challenges of the complex problem remain to be addressed:

i) Multiple control variables need to be coordinated for achieving the multiple objectives. Both the ventilation rate and zone mass flow rates require to be coordinated as to optimize energy efficiency for achieving thermal comfort and IAQ simultaneously. Particularly, a good IAQ requires high ventilation rate and zone mass flow rates. However, this may induce lower temperature bound violations and high energy cost.

ii) The problem is non-linear and non-convex due to the complex system dynamics. Both the energy cost and system dynamics (i.e., temperature and $\mathrm{CO}_{2}$ ) are nonlinear w.r.t the control inputs: zone mass flow rates and ventilation rate.

iii) There exist various spatial and temporal couplings. The optimal operation of HVAC systems should consider zone temperature and $\mathrm{CO}_{2}$ inertia, which correspond to substantial temporal constraints. Besides, the inter-zone heat transfer and the recirculated air to AHU induce tight spatial couplings.

\section{A. Contributions}

Motivated by the literature, this paper studies the control of multi-zone commercial HVAC systems to optimize the energy cost for maintaining thermal comfort and IAQ simultaneously. We make the following contributions to overcome the computational challenges.

(C1) We propose a two-level control method integrated with the upper level control (ULC) and the lower level control (LLC) by exploiting the problem structures.

(C2) While the ULC adopts an existing distributed method, we develop a distributed method for the LLC to achieve scalability and computation efficiency.
(C3) We demonstrate the method's performance in energy cost saving, human comfort (i.e., temperature and $\mathrm{CO}_{2}$ ) as well as computation efficiency through simulations.

Our two-level structure is motivated by the independent zone temperature and zone $\mathrm{CO}_{2}$ dynamics (but both subject to the control inputs), which makes it possible to tackle the two comfort indexes successively. Therefore, we decompose the problem into two levels: the ULC first computes the optimal zone mass flow rates to for maintaining zone thermal comfort with minimal energy cost. Successively, the LLC strategically regulates the computed zone mass flow rates from ULC and ventilation rate to achieve the desirable IAQ. Such two-level paradigm makes it computationally tractable to achieve the two comfort indexes simultaneously while preserving the nearoptimal energy cost. Moreover, the two-level structure favors computation efficiency as the LLC is only invoked when the $\mathrm{CO}_{2}$ upper bounds are to be violated. Particularly, as the ULC on thermal comfort has been comprehensively studied in our previous work [15] and thus adopted, we place our main focus on bringing in the two-level structure and the LLC.

The remainder of this paper is structured as below. In Section II, we review the related works. In Section III, we present the problem formulation. In Section IV, we discuss the two-level distributed method. In Section V, we evaluate the performance of the method via simulations. In Section VI, we briefly conclude this paper.

\section{RELATED WORKS}

Developing advanced control for HVAC systems to improve energy efficiency has been an edge issue along building automation over the past decades. Various methods have been studied and some comprehensive review can refer to [2, 3]. Significantly, these existing works have proved that substantial energy can be saved by deploying advanced HVAC control while not compromising human comfort. From the computation structure standpoint, these methods can be categorized into centralized methods [4] and decentralized methods [5]. Centralized approaches are usually developed for singleroom/zone cases and not amenable to commercial buildings due to the substantial computation burden (see [6, 7], for examples). By contrast, a number of decentralized approaches have been developed to address the computation challenges of commercial HVAC systems [5, 15, 16].

Nevertheless, one critical issue to be noticed is that most of these works have focused on thermal comfort (i.e., temperature, humidity, etc.) and lack IAQ management while pursuing energy cost savings (see $[17,18]$ and the references therein). In this backdrop, the IAQ may be awful and beyond tolerance though the temperature is maintained comfortable. For example, the pollutants and particles, especially $\mathrm{CO}_{2}$ concentration accumulate over the time if sufficient ventilation (i.e, outdoor fresh air) is not provided. This is easy to understand as high proportion of recirculated air is preferred to reduce the cooling load. In the literature, the IAQ management is mostly addressed by some simple ventilation rules referred to the demand controlled ventilation strategies (DCVs). Such methods could be $\mathrm{CO}_{2}$-based [19-21] or occupancy-based [22-24]. The 
main ideas are adjusting the amounts of fresh air infusion based on the detected instantaneous $\mathrm{CO}_{2}$ concentration or occupancy. Whereas for multi-zone commercial buildings, the DCVs tend to cause over-ventilation or under-ventilation due to zone $\mathrm{CO}_{2}$ or occupancy variations [25]. Another drawback is that the ventilation regulation for IAQ management are not coordinated with the thermal comfort, which will jeopardize the energy saving objective of HVAC systems.

It's imperative to jointly consider both thermal comfort and IAQ simultaneously both for achieving HVAC energy cost savings and maintaining human comfort. Such awareness has motivated the joint management of thermal comfort and IAQ for single-zone cases $[12,13,26]$. Particularly, most of these methods depend on simplified linear models to capture system dynamics. Therefore, the methods and modelings are generally not amenable to multi-zone commercial buildings. As a scarce exception, [14] studied both thermal comfort and IAQ management for commercial HVAC systems based on Lyapunov optimization. The performance of the method both in maintaining human comfort (i.e., thermal comfort and IAQ) and saving energy cost was demonstrated on a 4-zone case study. However, the computation tractability for larger scale applications remains to be addressed and motivates this work.

\section{Problem Formulation}

\section{A. HVAC Systems for Commercial Buildings}

The configuration for a typical commercial HVAC system is shown in Fig. 1, which mainly consists of an Air Handling Unit (AHU), Variable Air Volume (VAV) boxes and a chiller system (not shown here). The AHU is basically integrated with a damper, a cooling/heating coil and a supply fan. The heating/cooling coil cools down/heats up the mixed air (the mixture of outside fresh air and inside recirculated air) to the set-point temperature. The damper regulates the ventilation rates: the fraction of recirculated air $d_{r}$ or the fraction of outdoor fresh air $1-d_{r}$. Therefore, a smaller $d_{r}$ specifies higher proportion of fresh air infusion but induces higher cooling demand for the HVAC system, and vice versa. The supply fan is for driving the circulation of air within the building duct network. As another main component, the VAV boxes are connected to the zones, each of which consists of a damper and a heating coil. The damper regulates the zone mass flow rate and the heating coil reheats the supply air if necessary (not discussed in this paper). The chiller system is usually constituted by a chiller pump, water tank and the chiller, which provides continuous chilled water to the cooling coils of AHU. Except for the chiller, the chiller pump is also partially responsible for the HVAC's energy consumption for circulating the water between the water tank and the chiller. This paper studies HVAC systems with constant water flow system [27] where the chiller pump's energy consumption can be regarded as fixed and thus not explicitly discussed. Besides, without loss of generality, this paper studies the cooling mode. More details of the HVAC systems can refer to [4, 28].

As aforementioned, we seek to minimize the energy cost for maintaining both zone thermal comfort and IAQ simultaneously. In such setting, both zone air flow rates and ventilation

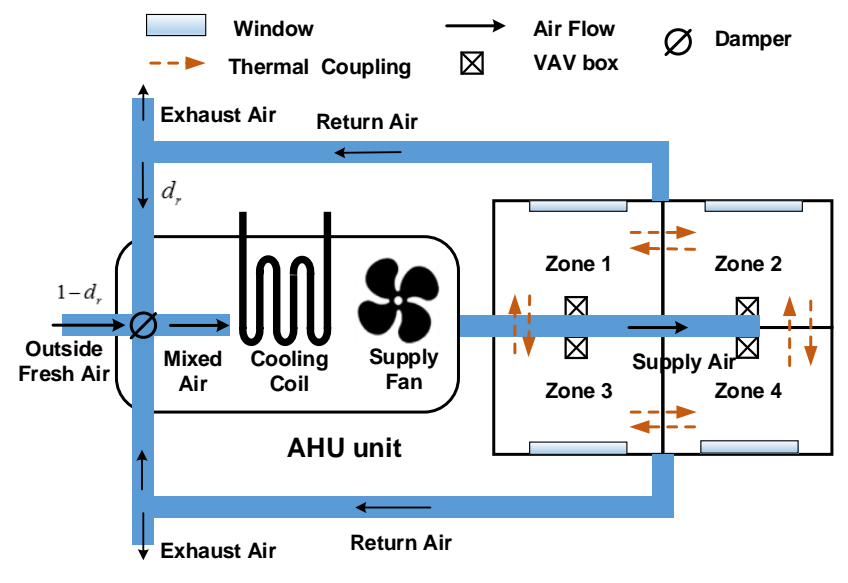

Fig. 1. The schematic of the HVAC system for multi-zone buildings.

rate $\left(d_{r}\right)$ need to be coordinated. The problem is studied in a discrete-time framework on a daily basis with $\Delta_{k}=30 \mathrm{~min}$ 's sampling and computing epoch. To account for the multi-stage control under uncertainties (e.g., weather, occupancy, etc.), we deploy the model predictive control (MPC) framework: the control inputs at each executed epoch are computed based on the predicted information over a look-ahead planning horizon $H=10(5 \mathrm{~h})$. This process is repeated with the time evolving until the end of the optimization horizon $\mathcal{T}$.

\section{B. Zone Thermal Dynamics}

We consider a commercial building with $I$ zones indexed by $\mathcal{I}=\{1,2, \cdots, I\}$. At each computing epoch, the zone thermal dynamics over the planning horizon $\mathcal{H}=\{0,1, \cdots, H-1\}$ are captured by a Resistance-Capacitance (RC) network [29, 30]:

$$
\begin{gathered}
C_{i}^{\rho}\left(T_{i}(k+1)-T_{i}(k)\right)=\sum_{j \in \mathcal{N}_{i}} \frac{T_{j}(k)-T_{i}(k)}{R_{i j}} \Delta_{k}+\frac{T_{o}(k)-T_{i}(k)}{R_{o i}} \Delta_{k} \\
\quad+c_{p} m_{i}^{z}(k)\left(T_{c}-T_{i}(k)\right) \Delta_{k}+Q_{i}(k) \Delta_{k}, \forall i \in \mathcal{I}, k \in \mathcal{H} .
\end{gathered}
$$

where $k \in \mathcal{H}$ and $i, j \in \mathcal{N}$ denote the time and zone indices. $C_{i}^{\rho}$ is the zone air heat capacity. $T_{i}(k), T_{o}(k)$ and $T_{c}$ denote the zone temperature, outdoor temperature and the set-point temperature of AHU, respectively. $R_{i j}\left(R_{j i}\right)$ denotes the adjacent zone thermal resistance. Particularly, $R_{o i}$ denotes the thermal resistance between zone $i$ and the outside, We use $\mathcal{N}_{i}$ to indicate the set of spatially adjacent zones to zone $i . c_{p}$ is the specific heat of the air. $m_{i}^{z}(k)$ indicates the zone mass flow rate. $Q_{i}(k)$ captures zone internal heat gains from the occupants and electrical equipment, which can estimated by the occupancy [31].

We translate (1) into a standard form:

$$
\begin{aligned}
& T_{i}(k+1)=A_{i i} T_{i}(k)+\sum_{j \in \mathcal{N}_{i}} A_{i j} T_{j}(k) \\
& \quad+C_{i i} m_{i}^{z}(k)\left(T_{i}(k)-T_{c}\right)+D_{i}(k), \quad \forall i \in \mathcal{I}, k \in \mathcal{H} .
\end{aligned}
$$

where $A_{i i}=1-\left(\sum_{j \in \mathcal{N}_{i}} \frac{\Delta_{k}}{R_{i j} C_{i}^{\rho}}+\frac{\Delta_{k}}{C_{i}^{\rho} R_{o i}}\right), A_{i j}=\frac{\Delta_{k}}{C_{i}^{\rho} R_{i j}}, C_{i i}=$ $-\frac{\Delta_{k} \cdot C_{p}}{C_{i}^{\rho}}$, and $D_{i}(k)=\frac{\Delta_{k} T_{o}(k)}{C_{i}^{\rho} R_{o i}}+\frac{\Delta_{k} \cdot Q_{i}(k)}{C_{i}^{p}}$. 
Similar to $[12,14]$, this paper uses $\mathrm{CO}_{2}$ concentration as an IAQ indicator. The zone $\mathrm{CO}_{2}$ dynamics for multi-zone commercial buildings can be described by [14]

$$
\begin{aligned}
m_{i}\left(C_{i}(k+1)\right. & \left.-C_{i}(k)\right)=N_{i}(k) C_{g} \Delta_{k} \\
& +m_{i}^{z}(k)\left(C_{z}(k)-C_{i}(k)\right) \Delta_{k} \forall i \in \mathcal{I}, k \in \mathcal{H}
\end{aligned}
$$

where $m_{i}$ denotes zone air mass. $C_{i}(k)$ (in ppm) denotes zone $\mathrm{CO}_{2}$ concentration. We suppose the occupants are the main source of $\mathrm{CO}_{2}$ generation and thus the $\mathrm{CO}_{2}$ accumulation can be estimated by the average $\mathrm{CO}_{2}$ generation rate per person $C_{g}\left(\mathrm{~g} \mathrm{~h}^{-1}\right)$ multiplied by the occupancy $N_{i}(k)$ as indicated in the first term of right-hand side. $C_{z}(k)$ denotes the $\mathrm{CO}_{2}$ concentration of supply air, which can be estimated by

$$
\begin{aligned}
& C_{z}(k)=\left(1-d_{r}(k)\right) C_{o}(k)+d_{r}(k) C_{\mathrm{m}}(k), \\
& \text { with } C_{\mathrm{m}}(k)=\frac{\sum_{i \in \mathcal{I}} m_{i}^{z}(k) C_{i}(k)}{\sum_{i \in \mathcal{I}} m_{i}^{z}(k)}, \forall k \in \mathcal{H}
\end{aligned}
$$

where $d_{r}(k) \in[0,1]$ denotes the fraction of return air delivered to AHU (i.e., the ventilation rate to be controlled). $C_{m}(k)$ characterizes the average $\mathrm{CO}_{2}$ concentration of the return air from all zones.

From (3) and (4), we note that the zone $\mathrm{CO}_{2}$ dynamics are non-linear and fully coupled through the recirculated air.

\section{Decision Variables}

Our objective is to minimize the HVAC energy cost for maintaining thermal comfort and IAQ. Therefore, our decision variables can be divided into control variables and state variables. The control variables are the control inputs of the HVAC system that affect indoor condition, which include the ventilation rate $d_{r}(k)$ and zone mass flow rate $m_{i}^{z}(k)$. Our state variables are zone temperature $T_{i}(k)$ and zone $\mathrm{CO}_{2}$ concentration $C_{i}(k)$, which indicate human comfort and requires to be maintained.

\section{Objective Function}

The energy consumption of the HVAC system is mainly incurred by the cooling coil $P_{f}(k)$ and supply fan $P_{f}(k)$ within AHU, i.e.,

$$
\begin{aligned}
P_{c}(k)= & c_{p} \eta\left(1-d_{r}(k)\right) \sum_{i \in \mathcal{I}} m_{i}^{z}(k)\left(T_{o}(k)-T_{c}\right) \\
& +c_{p} \eta d_{r}(k) \sum_{i \in \mathcal{I}} m_{i}^{z}(k)\left(T_{i}(k)-T_{c}\right) \\
P_{f}(k)= & \kappa_{f}\left(\sum_{i \in \mathcal{I}} m_{i}^{z}(k)\right)^{2}
\end{aligned}
$$

where $\eta$ is the reciprocal of the coefficient of performance (COP) of the chiller, which captures the ratio of provided cooling to the total consumed electrical power.

Considering the energy consumption is not easy to inspect in practice, we selected the total energy cost charged by the electricity price $c_{k}(\mathrm{~s} \$ / \mathrm{kW})$ on a daily basis as our objective:

$$
J=\sum_{k \in \mathcal{H}} c_{k}\left(P_{c}(k)+P_{f}(k)\right) \Delta_{k}
$$

\section{E. System Constraints}

The HVAC operation should respect the thermal comfort and IAQ requirements, which are represented by zone temperature bounds [5] and $\mathrm{CO}_{2}$ bounds [14] in this paper:

$$
\begin{gathered}
T_{i}^{\min } \leq T_{i}(k) \leq T_{i}^{\max }, \quad \forall i \in \mathcal{I}, k \in \mathcal{H} . \\
C_{i}(k) \leq C_{i}^{\max }, \quad \forall i \in \mathcal{I}, k \in \mathcal{H} .
\end{gathered}
$$

where $T_{i}^{\min }$ and $T_{i}^{\max }$ characterize the comfortable zone temperature ranges. $C_{i}^{\max }$ denotes the desirable zone $\mathrm{CO}_{2}$ upper bound. Particularly, the formulation can account for personalized human comfort by differentiating $T_{i}^{\min }, T_{i}^{\max }$ and $C_{i}^{\max }$ w.r.t the zones.

Additionally, the HVAC operation must abide by the physical limits of VAV boxes and AHU:

$$
\begin{gathered}
m_{i}^{z, \min } \leq m_{i}^{z}(k) \leq m_{i}^{z, \max }, \quad \forall i \in \mathcal{I}, k \in \mathcal{H} \\
\sum_{i \in \mathcal{I}} m_{i}^{z}(k) \leq m^{\max }, \quad \forall k \in \mathcal{H}
\end{gathered}
$$

where $m_{i}^{z, \min }$ and $m_{i}^{z, \max }$ denote the operation range of VAV boxes. $m^{\max }$ denotes the supply capacity of AHU.

Similarly, the damper of AHU for regulating ventilation rate is usually restricted to the operation range characterized by $d_{r}^{\min }$ and $d_{r}^{\max }$ to reduce wear and tear:

$$
d_{r}^{\min } \leq d_{r}(k) \leq d_{r}^{\max }, \quad \forall k \in \mathcal{H} .
$$

\section{F. The Problem}

Overall, the optimization problem at each computing epoch can be summarized as $(\mathcal{P})$.

$$
\begin{gathered}
\min _{\boldsymbol{m}_{i}^{z}, \boldsymbol{T}_{i}, \boldsymbol{C}_{i}, i \in \mathcal{I} . \boldsymbol{d}_{r}} J \\
\text { s.t. (2) }-(4),(7)-(8),(9)-(10),(11) .
\end{gathered}
$$

where we have $\boldsymbol{m}_{i}^{z}=\left[m_{i}^{z}(k)\right]_{k \in \mathcal{H}}, \boldsymbol{T}_{i}=\left[T_{i}(k)\right]_{k \in \mathcal{H}}$, $\boldsymbol{C}_{i}=\left[C_{i}(k)\right]_{k \in \mathcal{H}}(\forall i \in \mathcal{I})$ and $\boldsymbol{d}_{r}=\left[d_{r}(k)\right]_{k \in \mathcal{H}}$ denoting the concatenating decision variables over the computing epoch.

It's clear to see that problem $(\mathcal{P})$ is non-linear and nonconvex. The non-linearity and non-convexity both arise from the objective function and the constraints. Moreover, the substantial spatially and temporally coupled constraints imposed by zone temperature and zone $\mathrm{CO}_{2}$ makes it computationally intractable even for moderate commercial HVAC systems with centralized methods. Indeed, this motivates our two-level distributed method to be discussed.

\section{Two-Level Distributed Method}

To address the computational challenges of problem $(\mathcal{P})$, we propose a two-level distributed method (TLDM) based on the problem structures: the zone temperature and $\mathrm{CO}_{2}$ dynamics are independent (but both subject to the control inputs). We divide the problem into two levels: the upper level control (ULC) and the lower level control (LLC). The ULC is mainly responsible for thermal comfort and the LLC corresponds to IAQ management. The underlying idea of the two-level structure is to address the two comfort indexes successively while preserving the near-optimal energy saving 


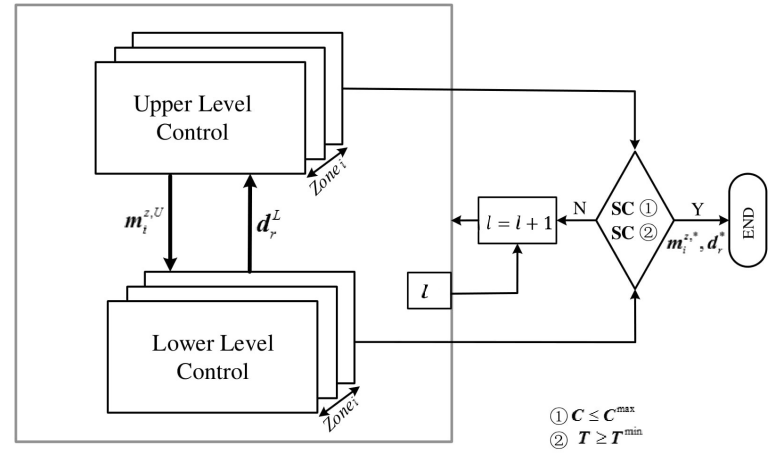

Fig. 2. The framework of two-level distributed method.

performance. Moreover, such two-level structure favors computation efficiency as the LLC requires to be invoked only if the $\mathrm{CO}_{2}$ is to be violated. Both the ULC and LLC use distributed implementation to achieve scalability and computation efficiency. We present the holistic framework of the TLDM in Fig. 2. Particularly, the ULC first computes the zone flow rates $\boldsymbol{m}^{z, U}$ for maintaining thermal comfort with minimal energy cost and then the LLC strategically regulates the ventilation rate $\boldsymbol{d}_{r}^{L}$ to achieve IAQ while preserving the near energy cost of ULC. The ULC and LLC alternate and communicate with each other. The implementation of ULC and LLC are as below.

\section{A. The Upper Level Control (ULC)}

The ULC focuses on maintaining zone thermal comfort with minimal energy cost. Considering that $i$ ) zone temperature is only affected by zone mass flow rate $m_{i}^{z}(k)$ and ii) the HVAC's energy cost is non-decreasing w.r.t. the ventilation rate $d_{r}(k)$, we define the ULC problem as

$$
\begin{array}{ll} 
& \min _{\boldsymbol{m}_{i}^{z}, \boldsymbol{T}_{i}, i \in \mathcal{I} .} J^{U} \\
\text { s.t. } & (2),(7),(9)-(10) . \\
& d_{r}(k)=d_{r}^{L}(k), k \in \mathcal{H} .
\end{array}
$$

where we have $J^{U}=J$ and $\boldsymbol{d}_{r}^{L}=\left[d_{r}^{L}(k)\right]_{k \in \mathcal{K}}$. The intuitive interpretation of ULC is to identify the zone mass flow rates for maintaining thermal comfort with minimal energy cost under the specified ventilation rate $d_{r}^{L}(k)$ from LLC (we can start with $d_{r}^{\max }(k \in \mathcal{H})$ ). For problem $\left(\mathcal{P}_{U}\right)$, there already exists a number of distributed methods $[5,15]$. We adopt our previously proposed distributed method [15] and place our main attention on the implementation of LLC.

\section{B. The Lower Level Control (LLC)}

As indicated in Fig. 2, the LLC only needs to be invoked when the zone $\mathrm{CO}_{2}$ concentrations violate the upper bounds (i.e., $\left.C_{i}(k) \geq C_{i}^{\max }\right)$. Clearly, both increasing zone mass flow rates and ventilation rate (decrease $d_{r}(k)$ ) can dilute zone $\mathrm{CO}_{2}$ thus improve IAQ. However, the latter is generally more expensive as it increase the cooling load of all zones. With the concern to preserve the near energy cost computed in ULC, our LLC adopts a two-phase method to achieve IAQ. We first seek to regulate zone mass flow rates to satisfy the user-defined zone $\mathrm{CO}_{2}$ bounds. To preserve the near energy cost of ULC, we select the deviation of zone mass flow rates relative to the ULC calculations as the objective. We have the following problem for the first phase of LLC:

$$
\begin{gathered}
\min _{\boldsymbol{m}_{i}^{z}, \boldsymbol{C}_{i}, i \in \mathcal{I} .} J^{L}=\sum_{k \in \mathcal{H}} \sum_{i \in \mathcal{I}}\left(m_{i}^{z}(k)-m_{i}^{z, U}(k)\right)^{2} \\
\text { s.t. } m_{i}^{z, U}(k) \leq m_{i}^{z}(k) \leq m_{i}^{z, \max }, \forall i \in \mathcal{I} . \\
(3)-(4),(8),(10) .
\end{gathered}
$$

where constraints (3)-(4) and (8) capture zone $\mathrm{CO} 2$ dynamics and bounds. Constraints (10) denotes the supply capacity of AHU. Particularly, constraints (12) model the zone mass flow rate lower bounds for maintaining thermal comfort, which is obtained from the ULC.

We suppose to achieve thermal comfort and IAQ simultaneously by solving problem $\left(\mathcal{P}_{L}\right)$, however things may not always happen in that way. We note that only the upper comfortable zone temperature bounds can be maintained but not necessarily the lower ones by (12). Therefore, it may occur that the desirable $\mathrm{CO}_{2}$ is achieved by solving problem $\left(\mathcal{P}_{L}\right)$ but the zone temperature drops beyond the lower bounds due to the substantial increase of zone mass flow rates. Actually, this means regulating zone mass flow rates alone is not viable to achieve the two comfort indexes simultaneously, and the second phase of LLC should be invoked to increase the ventilation rate (decrease $\boldsymbol{d}_{r}$ ). As illustrated in Fig. 2, such two phases alternate until we achieve the two comfort indexes. Suppose the two-phase structure gets well across, the remaining problem is about the implementation.

First phase of $L L C$ : Clearly, for the first phase, we require to solve the non-linear and non-convex problem $\left(\mathcal{P}_{L}\right)$, which is a nontrivial task. We note that the zone $\mathrm{CO}_{2}$ dynamics are fully coupled though the recirculated air, posing the primary challenge to develop a distributed method. To handle this, we introduce a learning framework to estimate $\mathrm{CO}_{2}$ concentration for the supply air $C_{z}(k)$. Therefore, the procedure to solve problem $\left(\mathcal{P}_{L}\right)$ contains two successive steps: first estimate $C_{z}(k)$ and then solve problem of $\left(\mathcal{P}_{L}\right)$ with the estimated $C_{z}(k)$. Specifically, with the estimated $\boldsymbol{C}_{z}(k)$, we have the decoupled zone $\mathrm{CO}_{2}$ dynamics:

$$
\begin{aligned}
C_{i}(k+1)=C_{i}(k)+ & E_{i}(k) m_{i}^{z}(k) \\
& +F_{i}(k) m_{i}^{z}(k) C_{i}(k)+G_{i}(k)
\end{aligned}
$$

where $E_{i}(k)=C_{z}(k) \Delta_{k} / m_{i}, \quad F_{i}(k)=-\Delta_{k} / m_{i}$ and $G_{i}(k)=N_{i}(k) C_{g} \Delta_{k} / m_{i}$ are now constant parameters.

We note that the zone $\mathrm{CO}_{2}$ dynamics (13) are bilinear w.r.t the zone mass flow rate $m_{i}^{z}(k)$ and zone $\mathrm{CO}_{2} C_{i}(k)$. To address the non-linearity, we introduce some auxiliary decision variables $Z_{i}(k)=m_{i}^{z}(k) C_{i}(k)$ and use the McCormick 
envelopes [32] to relax the bilinear terms:

$$
\begin{aligned}
& Z_{i}(0)=m_{i}^{z}(0) C_{i}(0) . \\
& Z_{i}(k) \geq m_{i}^{z, \min } C_{i}(k)+m_{i}^{z}(k) C_{i}^{\min }-m_{i}^{z, \min } C_{i}^{\min }, \\
& Z_{i}(k) \geq m_{i}^{z, \max } C_{i}(k)+m_{i}^{z}(k) C_{i}^{\max }-m_{i}^{z, \max } C_{i}^{\max }, \\
& Z_{i}(k) \leq m_{i}^{z}(k) C_{i}^{\max }+m_{i}^{z, \min } C_{i}(k)-m_{i}^{z, \min } C_{i}^{\max }, \\
& Z_{i}(k) \leq m_{i}^{z, \max } C_{i}(k)+m_{i}^{z}(k) C_{i}^{\min }-m_{i}^{z, \max } C_{i}^{\min }, \\
& \quad \forall k \in \mathcal{H} \backslash\{0\} .
\end{aligned}
$$

where $C_{i}(0)$ denotes the measured zone $\mathrm{CO}_{2}$ at the beginning of current computing epoch.

By invoking the auxiliary decision variables $Z_{i}(k)$, we have the following relaxed convex problem for problem $\left(\mathcal{P}_{L}\right)$ :

$$
\begin{gathered}
\min _{\boldsymbol{m}_{i}^{z}, \boldsymbol{C}_{i}, \boldsymbol{Z}_{i}, \forall i \in \mathcal{I} .} J^{L}=\sum_{k \in \mathcal{H}} \sum_{i \in \mathcal{I}}\left(m_{i}^{z}(k)-m_{i}^{z, U}(k)\right)^{2} \\
\text { s.t. } \quad \begin{aligned}
& C_{i}(k+1)=C_{i}(k)+E_{i}(k) m_{i}^{z}(k)+F_{i}(k) Z_{i}(k) \\
&+G_{i}(k), \quad \forall i \in \mathcal{I}, k \in \mathcal{H} . \\
& \text { (8), (10), (12), (13a)-(13e). }
\end{aligned}
\end{gathered}
$$

We note problem $\left(\mathcal{P}_{L}^{\prime}\right)$ is characterized by $\left.i\right)$ a decomposable objective function w.r.t. the zones and ii) coupled linear constraints. This problem can be efficiently tackled by the Accelerated Distributed Augmented Lagrangian (ADAL) method [33], which may be more clearly seen by recasting it into a standard form:

$$
\begin{array}{ll}
\min _{\boldsymbol{x}_{i}, i \in \mathcal{I}} J^{L}=\sum_{i \in \mathcal{I}} J_{i}^{L}\left(\boldsymbol{x}_{i}\right) \\
\text { s.t. } & \sum_{i=0}^{I} \boldsymbol{A}_{i}^{c} \boldsymbol{x}_{i}=\boldsymbol{b}^{c} . \\
& \boldsymbol{x}_{i} \in \mathcal{X}_{i}, \forall i \in \mathcal{I} \cup\{0\} .
\end{array}
$$

where $\boldsymbol{x}_{i}=\left[\left(\boldsymbol{x}_{i}(k)\right)^{T}\right]_{k \in \mathcal{H}}^{T}, \boldsymbol{x}_{i}(k)=\left(C_{i}(k), m_{i}^{z}(k), Z_{i}(k)\right)^{T}$ concatenating the decision variables of zone $i . J_{i}^{L}\left(\boldsymbol{x}_{i}\right)=$ $\sum_{k \in \mathcal{H}}\left(m_{i}^{z}(k)-m_{i}^{z, U}(k)\right)^{2}$ represents the local objective function of zone $i . \sum_{i=0}^{I} \boldsymbol{A}_{i}^{c} \boldsymbol{x}_{i}=\boldsymbol{b}^{c}$ accounts for the coupled linear constraints (10) with an additional slack variables $\boldsymbol{x}_{0}(k) \geq 0$ introduced at each stage (transform the inequality constraints to equality constraints). $\mathcal{X}_{i}(\forall i \in \mathcal{I})$ indicates the local constraints (8), (12), (13a)-(13e) corresponding to zone $i$ and particularly $\mathcal{X}_{0}=\left\{\boldsymbol{x}_{0} \mid \boldsymbol{x}_{0} \geq \mathbf{0}\right\}$. We have $\boldsymbol{A}_{i}^{c}=$ $\left(\begin{array}{ccccccc}0 & 1 & 0 & 0 & 0 & 0 & \cdots \\ 0 & 0 & 0 & 0 & 1 & 0 & \cdots \\ \cdots & \cdots & \cdots & \cdots & \cdots & \cdots & \cdots\end{array}\right) \in \mathbb{R}^{H \times 3 H} \quad(\forall i \in \mathcal{I})$, and $\boldsymbol{b}^{c}=\left(m^{\max }, m^{\max }, \cdots, m^{\max }\right)^{T} \in \mathbb{R}^{H}$.

Following the standard procedure of ADAL [33], we have the augmented Lagrangian function:

$$
\begin{aligned}
\mathbb{L}_{\rho}\left(\boldsymbol{x}_{0}, \boldsymbol{x}_{1}, \cdots, \boldsymbol{x}_{I}, \boldsymbol{\alpha}\right)= & \sum_{i \in \mathcal{I}} J_{i}^{L}+\boldsymbol{\alpha}^{T}\left(\sum_{i=0}^{I} \boldsymbol{A}_{i}^{c} \boldsymbol{x}_{i}-\boldsymbol{b}^{c}\right) \\
& +\frac{\rho}{2}\left\|\sum_{i=0}^{I} \boldsymbol{A}_{i}^{c} \boldsymbol{x}^{i}-\boldsymbol{b}^{c}\right\|^{2}
\end{aligned}
$$

where $\boldsymbol{\alpha}=\left(\alpha_{0}, \alpha_{1}, \cdots, \alpha_{H-1}\right)^{T}$ are Lagrangian multipliers. $\rho(\rho>0)$ is penalty parameter.
Therefore, we have the following primal problem with given Lagrangian multipliers $\alpha$ :

$$
\begin{aligned}
\min _{\boldsymbol{x}_{0} . \boldsymbol{x}^{i}, \forall i \in \mathcal{I}} & \mathbb{L}_{\rho}\left(\boldsymbol{x}_{0}, \boldsymbol{x}_{1}, \cdots, \boldsymbol{x}_{I}, \boldsymbol{\alpha}\right) \\
\text { s.t. } & \boldsymbol{x}_{i} \in \mathcal{X}_{i}, \quad \forall i \in \mathcal{I} . \\
& \boldsymbol{x}_{0} \geq \mathbf{0} .
\end{aligned}
$$

The main procedures of using ADAL to solve problem $\left(\mathcal{P}_{L}^{\prime \prime}\right)$ generally contain three steps: $i$ ) solving the primal problem (17), ii) updating the Lagrangian multipliers $\boldsymbol{\alpha}$, and iii) updating the penalty factor $\rho$. While the last two procedures are standard, we illustrate how to solve the primal problem $\left(\mathcal{P}_{L}^{\prime \prime}\right)$ in a distributed fashion. Specifically, we define $I+1$ agents, where Agent $1 \sim I$ correspond to the $I$ zones and Agent 0 is a virtual agent for managing the slack decision variable $\boldsymbol{x}_{0}$. At each iteration $q$, we have the local objective functions the agents:

$$
\begin{aligned}
& \mathbb{L}_{\rho}^{0}\left(\boldsymbol{x}_{0}, \boldsymbol{x}_{-0}^{q}, \boldsymbol{\alpha}\right)=\boldsymbol{\alpha}^{T} \boldsymbol{A}_{0}^{c} \boldsymbol{x}_{0}+\frac{\rho}{2}\left\|\boldsymbol{A}_{0}^{c} \boldsymbol{x}_{0}+\sum_{i=1}^{I} \boldsymbol{A}_{i}^{c} \boldsymbol{x}_{i}^{q}-\boldsymbol{b}^{c}\right\|^{2} \\
& \mathbb{L}_{\rho}^{i}\left(\boldsymbol{x}_{i}, \boldsymbol{x}_{-i}^{q}, \boldsymbol{\alpha}\right)=J_{i}^{L}+\boldsymbol{\alpha}^{T} \boldsymbol{A}_{i}^{c} \boldsymbol{x}_{i}+\frac{\rho}{2}\left\|\boldsymbol{A}_{i}^{c} \boldsymbol{x}_{i}+\sum_{j \in \mathcal{I} \cup\{0\}, j \neq i} \boldsymbol{A}_{j}^{c} \boldsymbol{x}_{j}^{q}-\boldsymbol{b}^{c}\right\|^{2},
\end{aligned}
$$$$
\forall i \in \mathcal{I} \text {. }
$$

It' clear that we can obtain the estimated zone mass flow rates $m_{i}^{z}(k)$ and zone $\mathrm{CO}_{2} C_{i}(k)$ by solving problem $\left(\mathcal{P}_{L}^{\prime \prime}\right)$. Afterward, we invoke the procedure to update the estimation of $C_{z}(k)$ according to (4).

Overall, we present the details to solve problem $\left(\mathcal{P}_{L}\right)$ based on ADAL in Algorithm 1. We use the superscripts $p$ and $q$ to denote the iteration corresponding to solving the relaxed problem $\left(\mathcal{P}_{L}^{\prime \prime}\right)$ and updating the estimation of $C_{z}(k)$, respectively. For notation, $\boldsymbol{x}^{q}=\left[\left(\boldsymbol{x}_{j}^{q}\right)^{T}\right]_{j \in \mathcal{I} \cup\{0\}}^{T}$ represent the augmented control and state trajectories for all agents while $\boldsymbol{x}_{-i}^{q}=\left[\left(\boldsymbol{x}_{j}^{q}\right)^{T}\right]_{j \in \mathcal{I}\{0\}, j \neq i}^{T}$ excluding Agent $i$. For the ADAL method, we uses the residual error of coupled constraints: $r_{q}(\boldsymbol{x})=\left\|\sum_{i=0}^{I} \boldsymbol{A}_{i}^{c} \boldsymbol{x}_{i}^{q}-\boldsymbol{b}^{c}\right\| \leq \epsilon^{\mathrm{in}}$ (C1) as the stopping criterion. For the estimation of $\mathrm{CO}_{2}$ concentration for the supply air, we evaluate the deviations of any two successive estimation: $\left\|\boldsymbol{C}_{z}^{p+1}-\boldsymbol{C}_{z}^{p}\right\| \leq \epsilon^{\text {out }}(\mathbf{C} 2)$, where $\epsilon^{\text {in }}$ and $\epsilon^{\text {out }}$ are small positive thresholds.

Recursive feasibility: Recall that the bilinear terms $Z_{i}(k)=$ $m_{i}^{z}(k) C_{i}(k)$ are relaxed in problem $\left(\mathcal{P}_{L}^{\prime}\right)\left(\right.$ or $\left.\left(\mathcal{P}_{L}^{\prime \prime}\right)\right)$, therefore the recursive feasibility remains to be addressed. We propose a heuristic method (see Algorithm 2) to recover a control input $\hat{\boldsymbol{x}}=\left[\left(\hat{\boldsymbol{x}}_{i}(k)\right)^{T}\right]_{k \in \mathcal{H}}^{T}$ from the optimal solution of problem $\left(\mathcal{P}_{L}^{\prime \prime}\right)$, which can ensure the recursive feasibility and near energy saving performance. The main idea is to preserve the computed zone mass flow rates: $\hat{m}_{i}^{z}(k)=m_{i}^{z, *}(k)$ $(\forall i \in \mathcal{I}, k \in \mathcal{H})$ as they determine the HVAC's energy cost. In such setting, the desirable $\mathrm{CO}_{2}$ at each executed epoch $t$ will be sustained. As the method is deployed in MPC framework, the desirable $\mathrm{CO}_{2}$ over the day (optimization horizon $\mathcal{T}$ ) will be achieved along the computing epoch. We illustrate the feasibility issue by induction. For notation, we indicate the real zone zone $\mathrm{CO}_{2}$ trajectories as $\left[C_{i, t}\right]_{t \in \mathcal{T}}(\forall i \in \mathcal{I})$, which suppose to be obtained at the end of optimization horizon $\mathcal{T}$. 


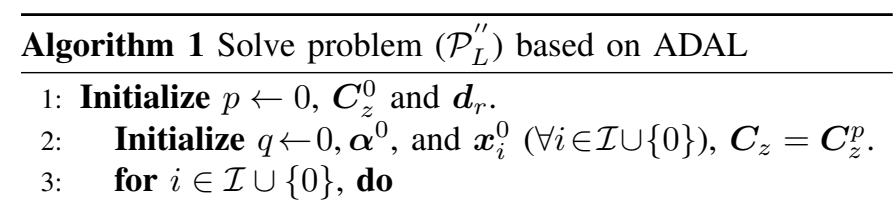

$$
\boldsymbol{x}_{i}^{q+1}=\arg \min _{\boldsymbol{x}_{i}} \mathbb{L}_{\rho}^{i}\left(\boldsymbol{x}_{i}, \boldsymbol{x}_{-i}^{q}, \boldsymbol{\alpha}^{q}\right) \text { s.t. } \boldsymbol{x}_{i} \in \mathcal{X}_{i} .
$$

4: $\quad$ end for

5: $\quad$ Update the Lagrangian multipliers:

$$
\boldsymbol{\alpha}^{q+1}=\boldsymbol{\alpha}^{q}+\rho\left(\sum_{i=0}^{I} \boldsymbol{A}_{i}^{c} \boldsymbol{x}_{i}^{q+1}-\boldsymbol{b}^{c}\right)
$$

6: If (C1) is satisfied, stop with $\boldsymbol{x}^{p}=\boldsymbol{x}^{q+1}$, otherwise $q \rightarrow q+1 \quad$ and go to Step 3 .

7: Estimate $\boldsymbol{C}_{z}^{p+1}$ according to

$$
\boldsymbol{C}_{z}^{p+1}=\left(1-\boldsymbol{d}_{r}\right) \boldsymbol{C}_{o}+\boldsymbol{d}_{r} \frac{\sum_{i \in \mathcal{I}} \boldsymbol{m}_{i}^{z, p} \boldsymbol{C}_{i}^{p}}{\sum_{i \in \mathcal{I}} \boldsymbol{m}_{i}^{z, p}} .
$$

8: If (C2) is satisfied, stop with $\boldsymbol{x}^{p}$, otherwise set $p \rightarrow p+1$ and go to Step 2.

Output: $\boldsymbol{x}^{*}=\left[\left(\boldsymbol{x}_{i}^{p}(k)\right)^{T}\right]_{k \in \mathcal{H}}^{T}, \boldsymbol{x}_{i}^{p}(k)=\left(C_{i}^{p}(k), m_{i}^{z, p}(k), Z_{i}^{p}(k)\right)^{T}$.

At each execution instant $t$, we can obtain the current zone $\mathrm{CO}_{2}$ measurements $C_{i}(0)=C_{i, t}(\forall i \in \mathcal{I})$. Suppose at time $t$ we have $C_{i, t} \leq C_{i}^{\max }(\forall i \in \mathcal{I})$, it suffices to address the feasibility issue by proving $C_{i, t+1} \leq C_{i}^{\max }(\forall i \in \mathcal{I})$ as below.

$$
\begin{aligned}
C_{i, t+1} & =C_{i}(1) \\
& =C_{i}(0)+E_{i}(0) \hat{m}_{i}^{z}(0)+F_{i}(0) \hat{m}_{i}^{z}(0) C_{i}(0)+G_{i}(0) \\
& =C_{i}(0)+E_{i}(0) m_{i}^{z, *}(0)+F_{i}(0) m_{i}^{z, *}(0) C_{i}(0)+G_{i}(0) \\
& =C_{i}(0)+E_{i}(0) m_{i}^{z, *}(0)+F_{i}(0) Z_{i}^{*}(0)+G_{i}(0) \\
& \leq C_{i}^{\max }, \forall i \in \mathcal{I} .
\end{aligned}
$$

where the first two equalities are directly from zone $\mathrm{CO}_{2}$ dynamics (13). The third and fourth equality are derived from $\hat{m}_{i}^{z}(k)=m_{i}^{z, *}(k)$ and (13a). The inequality is deduced from constraint (8).

Second phase of LLC: As discussed previously, it may occur that the lower comfortable zone temperature bounds may be violated for solving problem $\left(\mathcal{P}_{L}\right)$. In such situation, we are required to invoke the second phase of LLC to increase ventilation rate (decrease $d_{r}$ ). In principle, the regulating stepsize should be determined by the temperature violation amplitude, however it's difficult to coordinate such two different dimensions and we thus use a small constant step-size $\Delta d_{r}$. The overall framework of the proposed TLDM which embeds the ULC and LLC is presented in Algorithm 3 . We use $l$ to denote the iteration. Function $\mathbb{I}(A)$ is an indicator function, where we have $\mathbb{I}(A)=1$ with true condition $A$, otherwise $\mathbb{I}(A)=0$.

\section{Application}

We evaluate the performance of the TLDM on multi-zone commercial HVAC system via simulations. We first study the energy saving performance and computational advantage of the method via a benchmark (5 zones). After that the capability and scalability of the method to medium scale (10,20 zones) and large scale (50,100 zones) are illustrated.

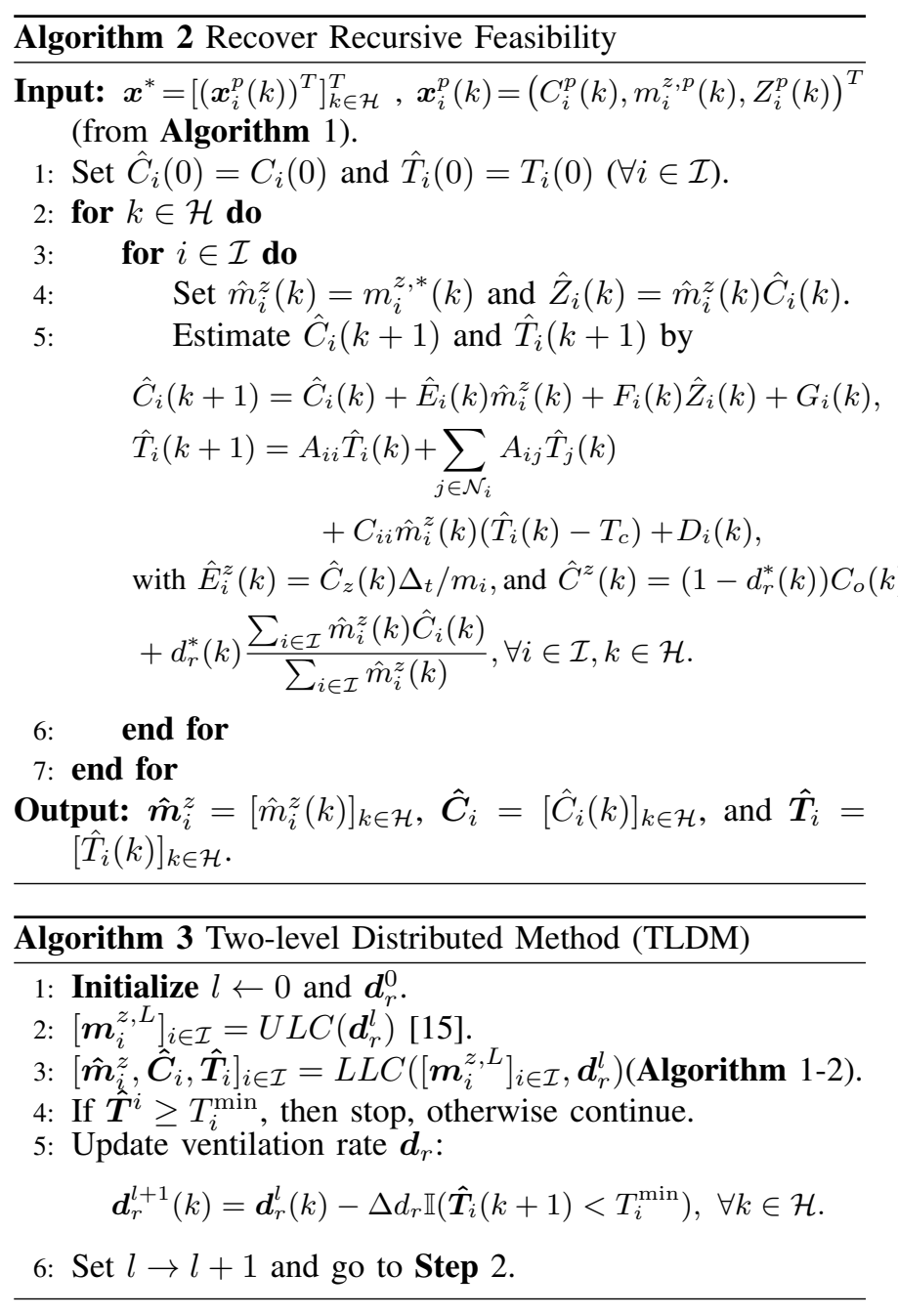

\section{A. Benchmark}

This part considers a 5-zone benchmark. We select the general comfortable zone temperature range $[24,26]^{\circ} \mathrm{C}$ and zone $\mathrm{CO}_{2}$ range $[0,800] \mathrm{ppm}$. The set-point temperature of AHU is $T_{c}=15^{\circ} \mathrm{C}$. We assume the zones are spatially next to each other $1 \leftrightarrow 2 \leftrightarrow 3 \leftrightarrow 4 \leftrightarrow 5 \leftrightarrow 1$. The initial zone temperature is set as $[29,30,31,30,29]^{\circ} \mathrm{C}$ (zone 1-5). The predicted outdoor temperature and zone occupancy are shown in Fig. 3. The HVAC's energy cost is calculated according to the time-of-use (TOU) price in Singapore [7]. The other parameters refer to TABLE I.

TABLE I

SimUlation PARAMETERS

\begin{tabular}{lll}
\hline Param. & Value & Units \\
\hline$C_{i}(i \in \mathcal{I})$ & $1.5 \times 10^{3}$ & $\mathrm{~kJ} \mathrm{~K}^{-1}$ \\
$c_{p}$ & 1.012 & $\mathrm{~kJ} / \mathrm{kg}-\mathrm{K}$ \\
$R_{o i}$ & 50 & $\mathrm{~kW} \mathrm{~K}^{-1}$ \\
$R_{i j}(i, j \in \mathcal{I})$ & 14 & $\mathrm{~kW} \mathrm{~K}^{-1}$ \\
$\kappa_{f}$ & 0.08 & - \\
$\eta$ & 1 & - \\
$C_{g}$ & 40 & $\mathrm{~g} \mathrm{~h}^{-1}$ \\
$\Delta d_{r}$ & 0.05 & - \\
$m_{i}^{z, \min }$ & 0 & $\mathrm{~kg} \mathrm{~h}^{-1}$ \\
$m_{i}^{z, \max }$ & 0.5 & $\mathrm{~kg} \mathrm{~h}^{-1}$ \\
\hline
\end{tabular}

In the benchmark, we compare the proposed method with $i$ ) distributed Token-based scheduling strategy (DTBSS) [5], ii) 

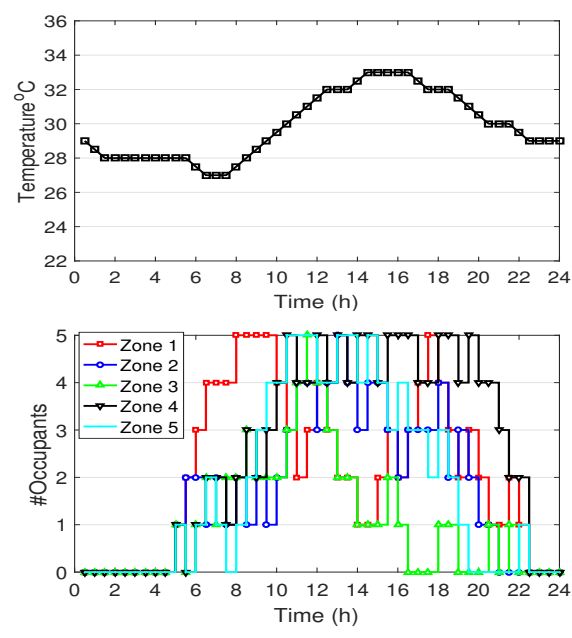

Fig. 3. (a) Outdoor temperature. (b) Zone occupancy.

centralized method, iii) the commonly-used DCV strategies $[25,34]$ and iv) sequential quadratic programming (SQP) [35]. Akin to most existing works, the DTBSS lacks IAQ management [5] and we therefore fix the ventilation rate in DTBSS as $d_{r}(k)=d_{r}^{\max }(\boldsymbol{k} \in \mathcal{H})$ to achieve the energy cost saving target. For the centralized method, we obtain the optimal solution by solving the non-linear optimization problem $(\mathcal{P})$ using the IPOPT solver embedded in MATLAB [36]. The DCVs calculate the amount of fresh air by the zone occupancy and zone area $[25,34]$, i.e.,

$$
m_{i}^{z \text {,fresh }}(k)=N_{i}(k) R_{p}+A_{i} R_{a}, \forall k \in \mathcal{H} .
$$

where $A_{i}$ denotes the area of zone $i . R_{p}$ and $R_{a}$ denote the average occupancy and space ventilation rate. This paper considers two DCVs: $i$ ) DCV I: calculating zone fresh air flow rates based on zone occupancy ( $\left.R_{p} \geq 0, R_{a}=0\right)$, and ii) DCV II: computing zone fresh air flow rates by zone occupancy and space $\left(R_{p} \geq 0, R_{a} \geq 0\right)$.

For single-zone case, the ventilation rate $\left(d_{r}\right)$ of DCVs can be straightforwardly determined by the fresh air infusion and mass flow rate. Nevertheless, for multi-zone case, the calculation of ventilation rate needs to account for zone diversity [25]:

$$
\begin{aligned}
& m^{z, \text { fresh }}(k)=\sum_{i \in \mathcal{I}} m_{i}^{z, \text { fresh }}(k), m^{z}(k)=\sum_{i \in \mathcal{I}} m_{i}^{z}(k), \\
& Z(k)=\max _{i \in \mathcal{I}}\left\{\frac{m_{i}^{z, \text { fresh }}(k)}{m_{i}^{z}(k)}\right\}, \quad X(k)=\frac{m^{z, \text { fresh }}(k)}{m^{z}(k)}, \\
& Y(k)=\frac{X(k)}{1+X(k)-Z(k)}, \quad d_{r}(k)=1-Y(k), \quad \forall k \in \mathcal{H} .
\end{aligned}
$$

One critical issue to be noticed is that the DCVs only account for IAQ by determining the amounts of fresh air infusion, the thermal comfort which corresponds to the zone mass flow rates is not considered. Therefore, we use the ULC of TLDM to calculate zone mass flow rates $m_{i}^{z}(k)$ in DCVs for fair comparisons. Therefore, similar to our TLDM, the DCVs correspond to two alternative procedures: obtain the zone mass flow rates by solving problem $\left(\mathcal{P}_{U}\right)$ (start with $d_{r}(k)=d_{r}^{\max }$ ) and amending the ventilation rate $d_{r}(k)$ according to (21).
TABLE II PERFORMANCE COMPARISONS

\begin{tabular}{lllllll}
\multicolumn{8}{c}{ PERFORMANCE COMPARISONS } \\
\hline Method & $R_{p}$ & $\begin{array}{l}R_{a} \\
(\mathrm{~L} / \mathrm{p})\end{array}$ & $\begin{array}{l}\text { Cost } \\
\left(\mathrm{L} / \mathrm{m}^{2}\right)\end{array}$ & $\begin{array}{l}\text { Time } \\
(\mathrm{s})\end{array}$ & TC & IAQ \\
\hline DTBSS & - & - & 245.03 & 2.11 & $\mathbf{Y}$ & $\mathbf{N}$ \\
Centralized & - & - & 247.15 & 575.07 & $\mathbf{Y}$ & $\mathbf{Y}$ \\
SQP & - & - & 275.91 & 151.26 & $\mathbf{Y}$ & $\mathbf{Y}$ \\
DCV I & 21 & 0 & 276.23 & - & $\mathbf{Y}$ & $\mathbf{Y}$ \\
DCV II & 16 & 0.04 & 274.99 & - & $\mathbf{Y}$ & $\mathbf{Y}$ \\
TLDM & - & - & 257.02 & 5.21 & $\mathbf{Y}$ & $\mathbf{Y}$ \\
\hline N=No, Y=Yes.
\end{tabular}
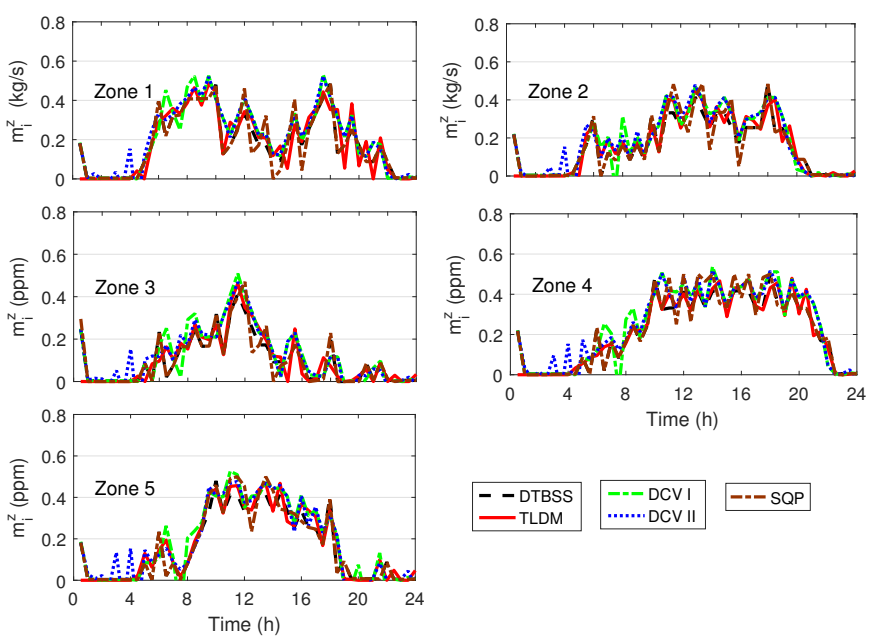

Fig. 4. Zone mass flow rate (Benchmark)

We present the energy cost, average computation time of each computing epoch ${ }^{1}$, satisfaction of thermal comfort (TC) and IAQ induced by the different methods in TABLE II. In particular, we exclude the DCVs while studying the computation time as they depend on off-line regulation for the occupancy and space ventilation rates, which is timeconsuming. We display the zone mass flow rates, zone temperature, zone $\mathrm{CO}_{2}$ and ventilation rate in Fig. 4-7. First of all, we find the zone thermal comfort is ensured by each method (Fig. 5) but not necessary for IAQ (Fig. 6) as we observe the desirable zone $\mathrm{CO}_{2}$ are out of range $[0,800] \mathrm{ppm}$ for DTBSS. This is not surprising as the DTBSS lacks IAQ management. Therefore, the method favors low ventilation rate (see Fig. 7) to save energy cost and fails to ensure IAQ. Further, we study the energy cost and computational efficiency of different methods. From TABLE II, we find the TLDM provides about $7.0 \%$ lower energy cost compared with the DCVs and the SQP. Since we observe close peaks over the zone temperature (Fig. 5) and zone $\mathrm{CO}_{2}$ (Fig. 6) trajectories for these methods, we conclude that the TLDM can maintain the same thermal comfort and IAQ but with less energy cost. As for the computational efficiency, the average computation time of TLDM for each executive epoch is about 5.21s (in parallel) with a slight increase over the DTBSS (2.11s). This is mainly attributed to the LLC to achieve IAQ. However, the TLDM obviously outperforms SQP in computation efficiency. While compared with centralized method, we imply the suboptimality of TLDM in energy cost is around $4 \%$ in the

${ }^{1}$ MATLAB R2016a on PC with Intel(R) Core(TM) i7-5500U CPU @2.40GHZ processor. 

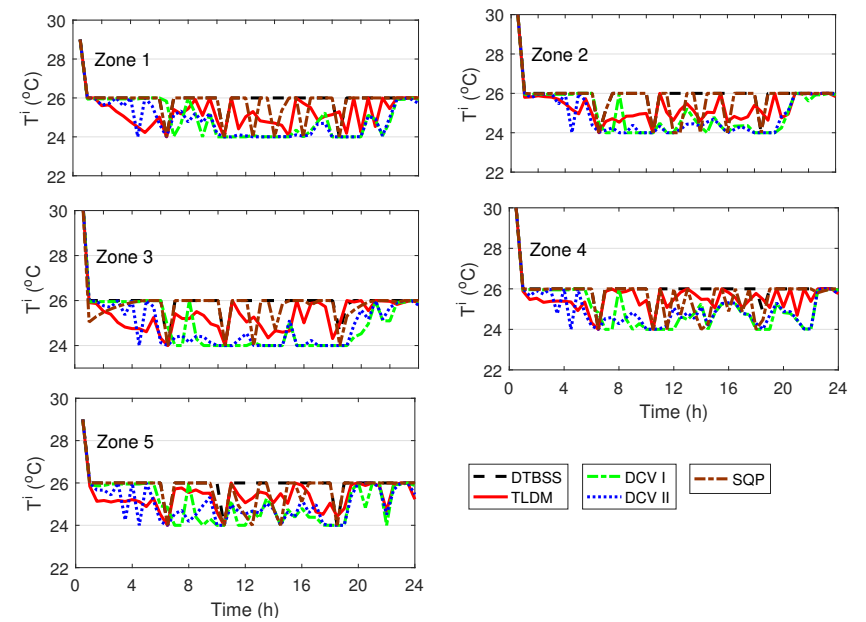

Fig. 5. Zone temperature (Benchmark).
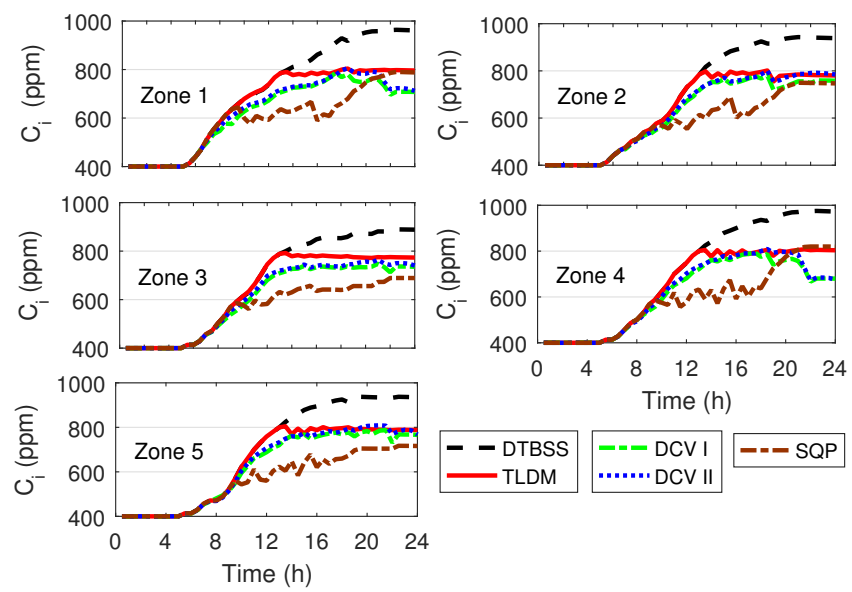

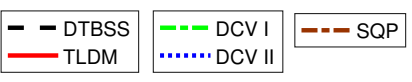

Fig. 6. Zone $\mathrm{CO}_{2}$ (Benchmark).

benchmark. Nevertheless, the computational benefit of TLDM is significant as the average computation time is reduced from $575.07 \mathrm{~s}$ to $5.21 \mathrm{~s}$.

\section{B. Scalability}

In this part, the proposed TLDM is applied to medium (10,20 zones) and large (50,100 zones) scale cases. Considering that centralized method and SQP are now computationally intractable, we compare the TLDM with the other three methods (i.e., DTBSS, and DCV I, II). For each case, we randomly generate a network to represent the spatial connectivity of the zones (the maximum number of adjacent zones zone is set as 4). In particular, the space and occupancy ventilation rates for the DCVs to achieve the IAQ are documented in TABLE III, which are obtained from off-line regulation. The other parameters refer to the benchmark in Section IV-A.

TABLE III

OCCUPANCY AND Space Ventilation Rates IN DCV I, II

\begin{tabular}{l|l|ll}
\hline \multirow{2}{*}{ \#zones } & DCV I & \multicolumn{2}{|c}{ DCV II } \\
\cline { 2 - 4 } 10 & $R_{p}(\mathrm{~L} / \mathrm{p})$ & $R_{p}(\mathrm{~L} / \mathrm{p})$ & $R_{a}\left(\mathrm{~L} / \mathrm{m}^{2}\right)$ \\
\cline { 2 - 4 } 20 & 19 & 15 & 0.03 \\
50 & 20 & 19 & 0.03 \\
100 & 21 & 19 & 0.03 \\
\hline
\end{tabular}

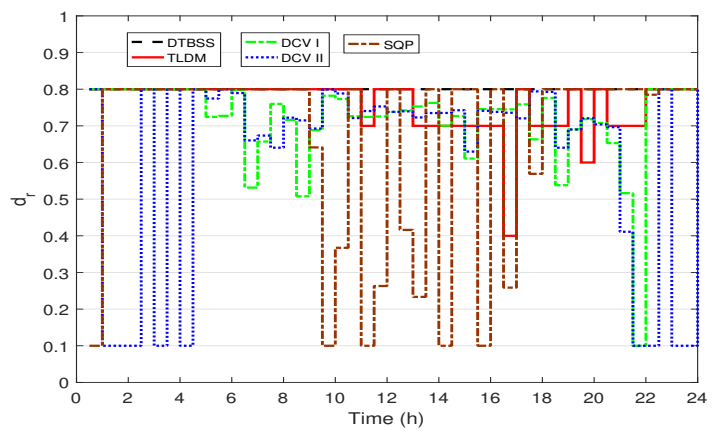

Fig. 7. Ventilation rate $\boldsymbol{d}_{r}$ (benchmark).

Similarly, we investigate the energy cost and computational efficiency of different methods, with the results shown in TABLE IV. Compared with the DTBSS, we observe a minor increase in energy cost and computation time with the TLDM to achieve IAQ. However, the TLDM is still computationally efficient and scalable by inspecting the average computation time (e.g., 21.68s for 100-zone case) versus the decision epoch 30mins. Besides, we observe similar gains of the TLDM over the DCVs in energy cost saving: $8.0-9.8 \%$ (DCV I) and $8.1-10.2 \%$ (DCV II). Moreover, except for the energy cost reduction, the TLDM is expected to be more applicable over the DCVs as it doesn't depend on time-consuming off-line regulation for the occupancy and space ventilation rate.

TABLE IV

PERFORMANCE COMPARISONS

\begin{tabular}{|c|c|c|c|c|c|c|c|c|}
\hline \multirow{3}{*}{ Method } & \multicolumn{6}{|c|}{ Medium } & \multirow{3}{*}{$\mathrm{TC}$} & \multirow{3}{*}{ IAQ } \\
\hline & \multicolumn{3}{|c|}{10} & \multicolumn{3}{|c|}{20} & & \\
\hline & $\begin{array}{l}\begin{array}{l}\text { Cost } \\
\text { (s\$) }\end{array}\end{array}$ & $\begin{array}{l}+- \\
(\%)\end{array}$ & $\begin{array}{l}\text { Time } \\
\text { (s) }\end{array}$ & $\begin{array}{l}\text { Cost } \\
(\mathrm{s} \$)\end{array}$ & $\begin{array}{l}+/- \\
(\%)\end{array}$ & $\begin{array}{l}\text { Time } \\
\text { (s) }\end{array}$ & & \\
\hline TLDM & 407.32 & - & 6.55 & 939.83 & - & 8.03 & $\mathbf{Y}$ & $\mathbf{Y}$ \\
\hline DTBSS & 387.07 & -4.97 & 2.35 & 887.12 & -5.61 & 2.71 & $\mathbf{Y}$ & $\mathbf{N}$ \\
\hline DCV I & 447.42 & +9.84 & - & 1015.50 & +8.05 & - & $\mathbf{Y}$ & $\mathbf{Y}$ \\
\hline DCV II & 440.42 & +8.13 & - & 1020.60 & +8.59 & - & $\mathbf{Y}$ & $\mathbf{Y}$ \\
\hline \multirow{3}{*}{ Method } & \multicolumn{6}{|c|}{ Large } & \multirow{3}{*}{$\mathrm{TC}$} & \multirow{3}{*}{ IAQ } \\
\hline & \multicolumn{3}{|c|}{50} & \multicolumn{3}{|c|}{100} & & \\
\hline & $\begin{array}{l}\text { Cost } \\
(\mathrm{s} \$) \times 10^{3}\end{array}$ & $\begin{array}{l}+- \\
(\%)\end{array}$ & $\begin{array}{l}\text { Time } \\
\text { (s) }\end{array}$ & $\begin{array}{l}\text { Cost } \\
(\mathrm{s} \$) \times 10^{3}\end{array}$ & $\begin{array}{l}+- \\
(\%)\end{array}$ & $\begin{array}{l}\text { Time } \\
\text { (s) }\end{array}$ & & \\
\hline TLDM & 2.65 & - & 13.28 & 5.80 & - & 21.68 & $\mathbf{Y}$ & $\mathbf{Y}$ \\
\hline DTBSS & 2.54 & -4.15 & 4.47 & 5.49 & -5.34 & 6.90 & $\mathbf{Y}$ & $\mathbf{N}$ \\
\hline DCV I & 2.91 & +9.81 & - & 6.31 & +8.79 & - & $\mathbf{Y}$ & $\mathbf{Y}$ \\
\hline DCV II & 2.92 & +10.19 & - & 6.36 & +9.66 & - & $\mathbf{Y}$ & $\mathbf{Y}$ \\
\hline
\end{tabular}

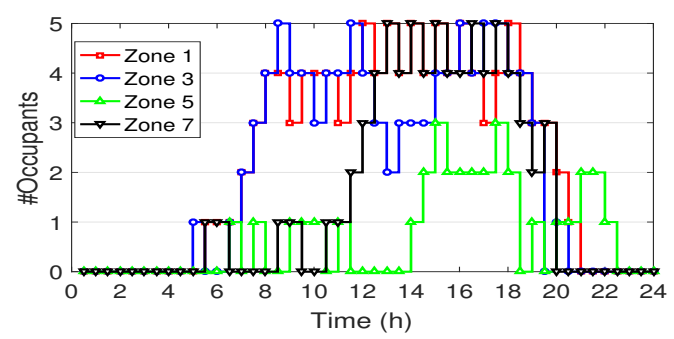

Fig. 8. The dynamic occupancy for the three zones among $I=50$ zones.

As an instance, we further investigate the 50-zone case study. We display the zone occupancy, zone mass flow rates, zone temperature, and zone $\mathrm{CO}_{2}$ for three randomly selected zones in Fig. 8-11. From Fig. $10-11$, we see both the TLDM 

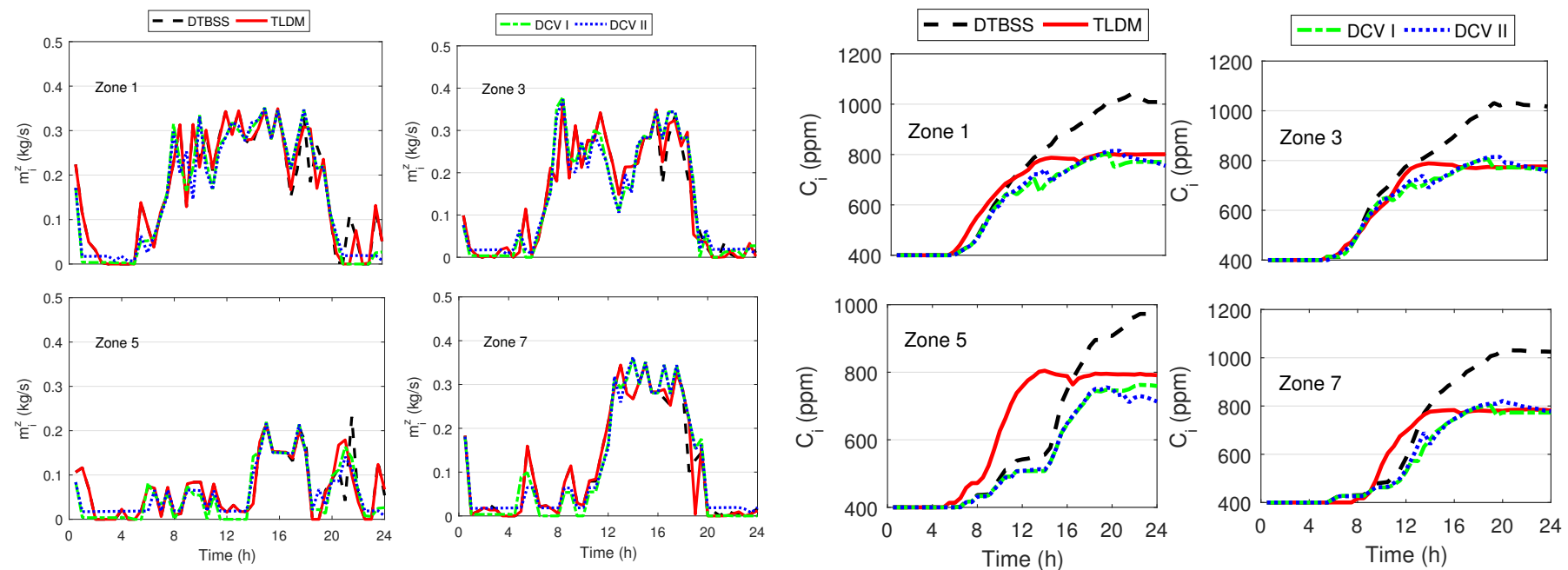

Fig. 9. The zone air flow rates for the three zones among $I=50$ zones.
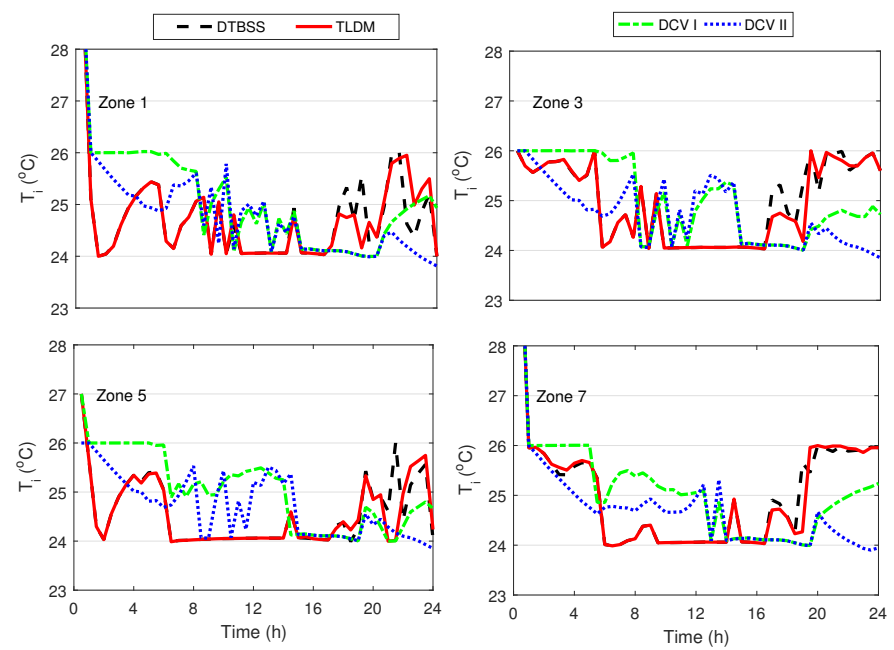

Fig. 11. The $\mathrm{CO}_{2}$ concentration for the four zones among $I=50$ zones

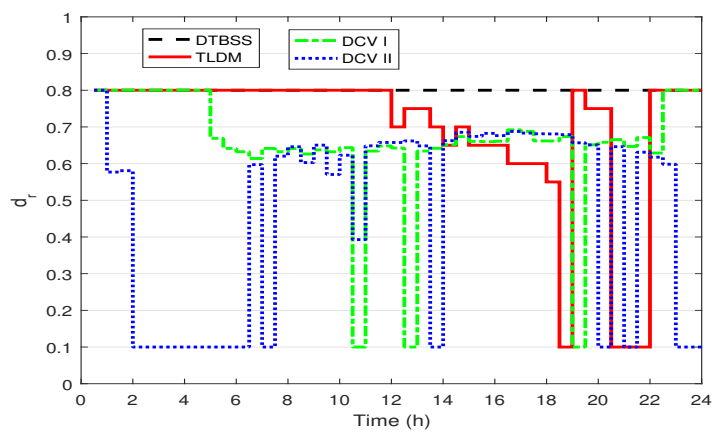

Fig. 12. The ventilation rate $\left(\boldsymbol{d}_{r}\right)$ of the HVAC system

Fig. 10. The temperature for the four zones among $I=50$ zones.

and the DCVs maintain the comfortable zone temperature range $\left([24,26]^{\circ} \mathrm{C}\right)$ and zone $\mathrm{CO}_{2}$ range $([0,800] \mathrm{ppm})$. Besides, we observe close peak ( $800 \mathrm{ppm})$ over the zone $\mathrm{CO}_{2}$ trajectories under the TLDM and the DCVs, which implies the close IAQ maintained by these methods. Besides, from Fig. 10 , we can have some insights in the characteristics of the TLDM to achieve thermal comfort and IAQ. Specifically, we see the zone temperature approaches the lower bound during the working hours with high occupancy. This phenomenon is caused by the two-phase structure of LLC in TLDM where the zone mass flow rates is first adjusted to achieve IAQ and then the ventilation rate is regulated if necessary.

We also compare the ventilation rates $\left(\boldsymbol{d}_{r}\right)$ of different methods in Fig. 12, which exhibit some interesting phenomenon to be interpreted. First of all, we see the ventilation rate of TLDM corresponds well to the occupancy. Specifically, we see relatively low ventilation rate (larger $\boldsymbol{d}_{r}$ ) during the off-working hours but the opposite over the working hours (smaller $\boldsymbol{d}_{r}$ ). This is reasonable as the LLC is only invoked to regulate the ventilation rate if the $\mathrm{CO}_{2}$ is to be violated, which generally results from high occupancy. Surprisingly, the results for DCV I and DCV II are almost opposite and quite different

from the TLDM. Specifically, we see that the ventilation rate $\left(\boldsymbol{d}_{r}\right)$ of DCV I corresponds well to the occupancy whereas the DCV II presents the opposite. These phenomenon are attributed to the rules that used to determine zone fresh air flow rates in the DCVs as discussed before. For DCV I, the zone fresh air totally depends on zone occupancy and thus we observe a synchronous pace of ventilation rate $\left(\boldsymbol{d}_{r}\right)$ with the occupancy. However, for DCV II, the zone mass flow rates are jointly determined by the occupancy and space. Therefore, during the off-working hours with low occupancy, we can observe higher ventilation rate as the proportions of zone fresh air flow rates dominate in the total zone mass flows rates (higher proportion) for maintaining thermal comfort.

\section{CONCLUSION}

This paper studied scalable control of multi-zone HVAC systems with the objective to reduce the energy cost for maintaining thermal comfort and IAQ simultaneously. This problem is computationally challenging due to the complex system dynamics. To cope with the difficulties, we proposed a two-level distributed method (TLDM) which integrates the upper and lower level control by exploiting the problem structures. Specifically, the upper level first optimizes zone mass flow rates to satisfy thermal comfort with minimal energy cost and the lower level regulates zone mass flow rates and the ventilation rate to achieve IAQ while preserving 
the near energy saving performance of ULC. As both the upper and lower level control use distributed computation, the proposed method is computationally efficient and scalable. The method's performance in energy saving and scalability was demonstrated by numeric studies. The sub-optimality of the proposed method in energy cost is around $4 \%$ but with significant computational benefits over the centralized method. Compared to the distributed Token-based scheduling strategy (DTBSS), the proposed method induces a marginal increase of energy costs but provides IAQ. In addition, the proposed method provided $8-10 \%$ energy savings over the demand controlled ventilation strategies (DCVs).

\section{REFERENCES}

[1] K. Ku, J. Liaw, M. Tsai, and T. Liu, "Automatic control system for thermal comfort based on predicted mean vote and energy saving," IEEE Transactions on Automation Science and Engineering, vol. 12, no. 1, pp. 378-383, 2015.

[2] H. Mirinejad, K. C. Welch, and L. Spicer, "A review of intelligent control techniques in HVAC systems," in 2012 IEEE Energytech, pp. 1-5, IEEE, 2012.

[3] A. Afram and F. Janabi-Sharifi, "Theory and applications of HVAC control systems-a review of model predictive control (MPC)," Building and Environment, vol. 72, pp. 343-355, 2014.

[4] A. Kelman and F. Borrelli, "Bilinear model predictive control of a HVAC system using sequential quadratic programming," in Ifac world congress, vol. 18, pp. 9869 9874, 2011.

[5] N. Radhakrishnan, Y. Su, R. Su, and K. Poolla, "Token based scheduling for energy management in building HVAC systems," Applied energy, vol. 173, pp. 67-79, 2016.

[6] Y. Yang, G. Hu, and C. J. Spanos, "Stochastic optimal control of HVAC system for energy-efficient buildings," arXiv preprint arXiv:1911.00840, 2019.

[7] Z. Xu, G. Hu, C. J. Spanos, and S. Schiavon, "Pmvbased event-triggered mechanism for building energy management under uncertainties," Energy and Buildings, vol. 152, pp. 73-85, 2017.

[8] H. Dasi, F. Xiaowei, and C. Daisheng, "On-line control strategy of fresh air to meet the requirement of IAQ in office buildings," in 2010 5th IEEE Conference on Industrial Electronics and Applications, pp. 845-848, IEEE, 2010.

[9] W. Li, C. Koo, S. H. Cha, T. Hong, and J. Oh, "A novel real-time method for HVAC system operation to improve indoor environmental quality in meeting rooms," Building and Environment, vol. 144, pp. 365-385, 2018.

[10] "Good indoor air quality leads to good decisions." https://www.vaisala.com/sites/default/files/documents/ VIM-G-HVAC-Good-Indoor-Air-Quality-Application-note-B pdf, 2015. Accessed Dec. 13, 2020.

[11] "Why measure $\mathrm{CO} 2$ in HVAC applications?" $\quad$ https://www.co2meter.com/blogs/news/ why-measure-co2-hvac-applications, 2020. Accessed Dec. 13, 2020.
[12] A. Parisio, M. Molinari, D. Varagnolo, and K. H. Johansson, "A scenario-based predictive control approach to building HVAC management systems," in 2013 IEEE International Conference on Automation Science and Engineering (CASE), pp. 428-435, IEEE, 2013.

[13] A. Parisio, D. Varagnolo, M. Molinari, G. Pattarello, L. Fabietti, and K. H. Johansson, "Implementation of a scenario-based MPC for HVAC systems: an experimental case study," IFAC Proceedings Volumes, vol. 47, no. 3, pp. 599-605, 2014.

[14] L. Yu, D. Xie, C. Huang, T. Jiang, and Y. Zou, "Energy optimization of hvac systems in commercial buildings considering indoor air quality management," IEEE Transactions on Smart Grid, vol. 10, no. 5, pp. 5103-5113, 2018.

[15] Y. Yang, G. Hu, and C. J. Spanos, "HVAC Energy cost optimization for a multi-zone building via a decentralized approach.” https://arxiv.org/abs/1905.10934, 2019. Accessed Feb. 17, 2020.

[16] J. Mei and X. Xia, "Distributed control for a multievaporator air conditioning system," Control Engineering Practice, vol. 90, pp. 85-100, 2019.

[17] C. C. Okaeme, S. Mishra, and J. T.-Y. Wen, "Passivitybased thermohygrometric control in buildings," IEEE Transactions on Control Systems Technology, vol. 26, no. 5, pp. 1661-1672, 2017.

[18] C. C. Okaeme, S. Mishra, and J. T. Wen, "A comfort zone set-based approach for coupled temperature and humidity control in buildings," in 2016 IEEE International Conference on Automation Science and Engineering (CASE), pp. 456-461, IEEE, 2016.

[19] N. Nassif, S. Kajl, and R. Sabourin, "Ventilation control strategy using the supply $\mathrm{CO} 2$ concentration setpoint," Hvac\&R Research, vol. 11, no. 2, pp. 239-262, 2005.

[20] T. R. Nielsen and C. Drivsholm, "Energy efficient demand controlled ventilation in single family houses," Energy and buildings, vol. 42, no. 11, pp. 1995-1998, 2010.

[21] A. Hesaraki, J. A. Myhren, and S. Holmberg, "Influence of different ventilation levels on indoor air quality and energy savings: A case study of a single-family house," Sustainable cities and society, vol. 19, pp. 165-172, 2015.

[22] Z. Wang and L. Wang, "Intelligent control of ventilation system for energy-efficient buildings with co2 predictive model," IEEE Transactions on Smart Grid, vol. 4, no. 2, pp. 686-693, 2013.

[23] N. Nassif, "A robust CO2-based demand-controlled ventilation control strategy for multi-zone HVAC systems," Energy and buildings, vol. 45, pp. 72-81, 2012.

[24] M. Marinov, T. Djamiykov, B. Ganev, and V. Zerbe, "Sensor-based multi-zone demand-controlled ventila-B21 1t681EN.

[25] K. Shan, Y. Sun, S. Wang, and C. Yan, "Development and in-situ validation of a multi-zone demand-controlled ventilation strategy using a limited number of sensors," Building and environment, vol. 57, pp. 28-37, 2012.

[26] A. Parisio, D. Varagnolo, D. Risberg, G. Pattarello, 
M. Molinari, and K. H. Johansson, "Randomized model predictive control for HVAC systems," in Proceedings of the 5th ACM Workshop on Embedded Systems For Energy-Efficient Buildings, pp. 1-8, ACM, 2013.

[27] Hattersley, "Variable flow vs constant flow." https://www. hattersley.com/page/hvac/variable-flow-systems. Accessed Dec. 20, 2019.

[28] X. Zhang, W. Shi, B. Yan, A. Malkawi, and N. Li, "Decentralized and distributed temperature control via HVAC systems in energy efficient buildings," arXiv preprint arXiv:1702.03308, 2017.

[29] Y. Lin, T. Middelkoop, and P. Barooah, "Issues in identification of control-oriented thermal models of zones in multi-zone buildings," in Decision and Control (CDC), 2012 IEEE 51st Annual Conference on, pp. 6932-6937, IEEE, 2012.

[30] M. Maasoumy, A. Pinto, and A. Sangiovanni-Vincentelli, "Model-based hierarchical optimal control design for HVAC systems," in ASME 2011 Dynamic Systems and Control Conference and Bath/ASME Symposium on Fluid Power and Motion Control, pp. 271-278, American Society of Mechanical Engineers, 2011.

[31] I. Korolija, L. Marjanovic-Halburd, Y. Zhang, and V. I. Hanby, "Influence of building parameters and hvac systems coupling on building energy performance," Energy and Buildings, vol. 43, no. 6, pp. 1247-1253, 2011.

[32] G. P. McCormick, "Computability of global solutions to factorable nonconvex programs: Part I-convex underestimating problems," Mathematical programming, vol. 10, no. 1, pp. 147-175, 1976.

[33] N. Chatzipanagiotis, D. Dentcheva, and M. M. Zavlanos, "An augmented lagrangian method for distributed optimization," Mathematical Programming, vol. 152, no. 1-2, pp. 405-434, 2015.

[34] Z. Sun, S. Wang, and Z. Ma, "In-situ implementation and validation of a $\mathrm{CO} 2$-based adaptive demand-controlled ventilation strategy in a multi-zone office building," Building and Environment, vol. 46, no. 1, pp. 124-133, 2011.

[35] J. Nocedal and S. J. Wright, "Sequential quadratic programming," Numerical optimization, pp. 529-562, 2006.

[36] Y. Kawajir, C. Laird, and A. Wachter, "Introduction to IPOPT: A tutorial for downloading, installing, and using IPOPT," 2006. 